\title{
Simulation of Cell Dielectric Properties Based on COMSOL
}

\author{
Shudong Li ${ }^{1}$, Xiaoyan Chen ${ }^{1 *}$, Fengze Han ${ }^{1}$ \\ ${ }^{1}$ College of Electronic Information and Automation, \\ Tianjin University of Science and Technology, China; \\ E-mail: *cxywxr@tust.edu.cn \\ www.tust.edu.cn
}

\begin{abstract}
The dielectric properties of cells can be observed by injecting a low amplitude current at different frequencies $(1 \mathrm{MHz} 100 \mathrm{MHz})$. The simulation research is taken on the software platform named COMSOL Multiphysics. The electric field and the cell model is created with prior information. By simulation, it's verified that at low frequencies, the region of interest (ROI) behaves the conductivity characteristic while the electrical signal cannot pass through the cell membrane due to its capacitor properties. With the excitation frequency increasing, the ROI behaves more permittivity characteristic that the current flowing through the cell membrane becomes more and the current density increases. The research of the cell dielectric properties provides an auxiliary method to diagnose the status of the cell.
\end{abstract}

Keywords: dielectric properties, cell model, excitation frequency, finite element method (FEM), auxiliary method

\section{Introduction}

\subsection{Cell structure}

From the biological point, cells are the smallest units that constitute the biological tissues. Most biological cells consist of cell fluid and membrane. The cell fluid can be divided into intracellular fluid and extracellular fluid as shown in Fig.1. Intracellular fluid can be regard as saline solution which consists of water mainly, inorganic salt and other metabolites. Extracellular fluid provides the living environment for cells with appropriate $\mathrm{PH}$ value and saline concentration. Both intracellular and extracellular fluid have good electrical conductivity ${ }^{[1]}$.

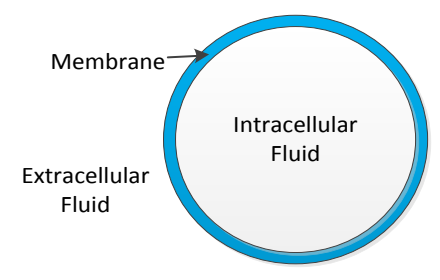

Fig.1. Simplified single cell structure model
The cell membranes are composed of insulating bilayer phospholipid and proteins. The membranes separate the intracellular and extracellular fluid. However, water and some micro-molecular proteins can exchange between intracellular and extracellular fluid, due to the permeable selective characteristic and the structure of the membranes.

\subsection{Cell dielectric properties}

The specified structure of a cell determines its special electrical characteristics. When a direct current or a low frequency alternating current is injected into the cell, a little or even none current can flow into the cell because of the insulated membrane. This behavior is called low-frequency dielectric property. When the excitation frequency gradually increasing, the membrane becomes electrically invisible and the current can inject into the cell by passing through the membrane. This performance is called high-frequency dielectric property ${ }^{[2]}$. The current lines flow as shown in Fig.2. 


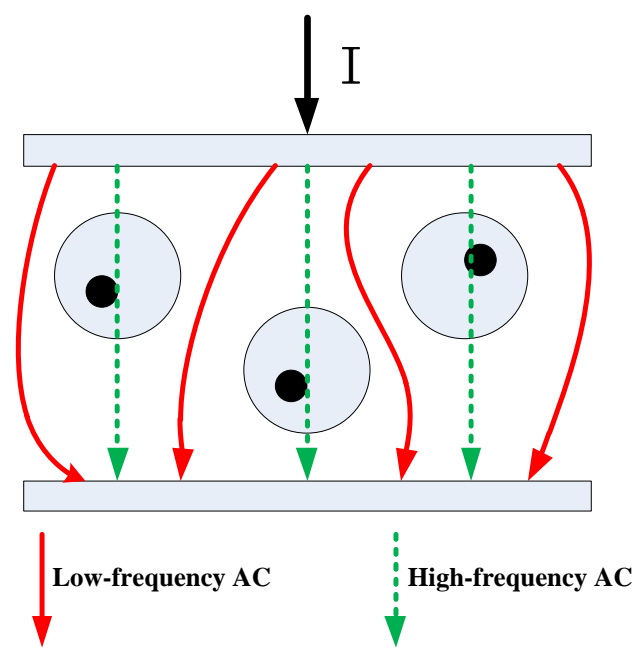

Fig.2. Current flows through the cell

According to the theory of cell dielectric properties, cell membrane has the characteristic of capacitance and the two cell fluids has the characteristic of resistance ${ }^{[3]}$. The electrical characteristics of single cell can be equaled to an electrical circuit as shown in Fig.3(a), where the parameters represent different parts of the cell.

$R_{m}$ : equivalent resistance of membrane

$C_{m}$ : equivalent capacitance of membrane

$R_{i}$ : equivalent resistance of intracellular fluid

$C_{i}$ : equivalent capacitance of intracellular fluid

$R_{e}$ :equivalent resistance of extracellular fluid

$C_{e}$ : equivalent capacitance of extracellular fluid

While $R_{m}, C_{i}$ and $C_{e}$ are much larger than $C_{m}, R_{i}$ and $R_{e}$, less current would go through them, so these can be neglected to achieve a simplified equivalent electrical model as shown in Fig.3(b).

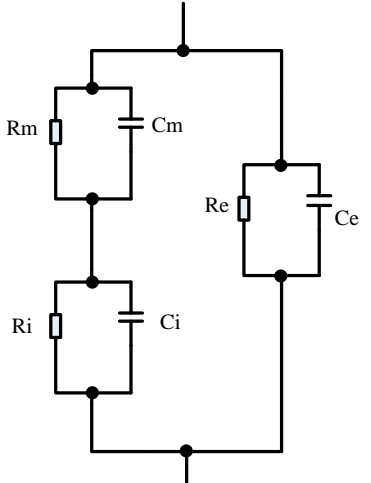

(a)

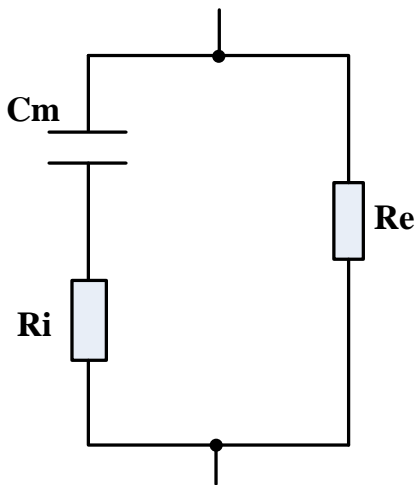

(b)
Fig.3(a). A equivalent circuit model of single cell (b). Simplified equivalent circuit model of single cell

\subsection{Finite element method}

The finite element method (FEM) was defined and published by Clough in 1960 . With the development of computer, the numerical solution of differential equations has been developed and applied a lot. The main idea of FEM is to approach an entire continuous domain by finite discrete domains. The unknown function on the entire domain is represented by functions on each discrete domain approximately ${ }^{[4]}$. A commercial software named COMSOL Multiphysics is a powerful solution engine.

\section{Method}

This section uses the software COMSOL Multiphysics to build the model of single cell. The geometric shape of model and the value of dielectric properties of cell are selected referring to blood cells ${ }^{[5]}$.

\subsection{Single cell model}

\subsubsection{Modeling and parameter settings}

The parameters of the single cell model is shown in Fig.4. The meaning of the parameters is as follows:

$\varepsilon_{\mathrm{e}}$ : permittivity of extracellular fluid

$\sigma_{e}$ : conductivity of extracellular fluid

$\varepsilon_{i}$ : permittivity of intracellular fluid

$\sigma_{\mathrm{i}}$ : conductivity of intracellular fluid

$\varepsilon_{\mathrm{m}}$ : permittivity of membrane

$\sigma_{\mathrm{m}}$ : conductivity of membrane

$\mathrm{R}$ : radius of single cell

$\mathrm{T}_{\mathrm{m}}$ : thickness of membrane

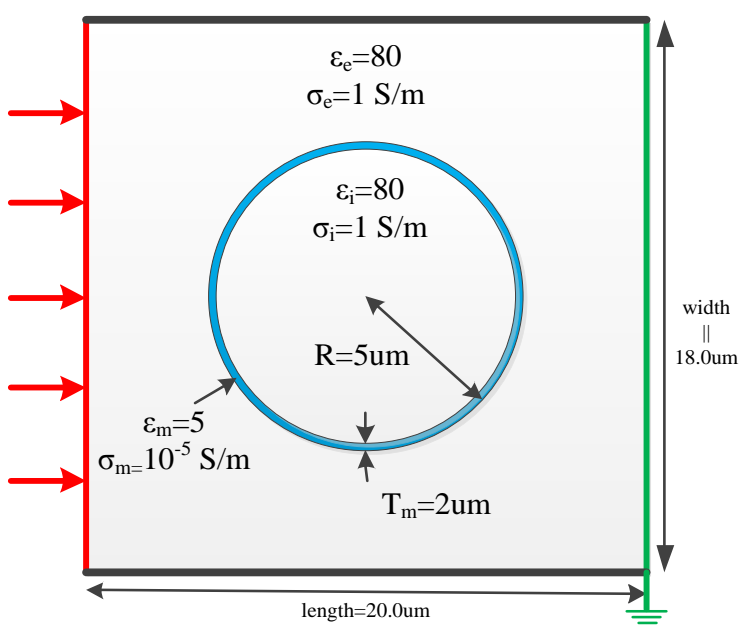

Fig.4. Geometric and electrical parameters of single cell model 
As shown in Fig.4, the excitation current with current density of $1 \mathrm{~A} / \mathrm{m}^{2}$ enters the region of interest (ROI) from the left boundary and flows out from the right boundary. The above and bottom boundaries are electrical insulation.

\subsubsection{Meshing grid}

Meshing is the process by which the active geometrical space (domain) of a model is sub-divided into a collection of sufficiently smaller spaces so that linear or higher order polynomial approximations can be used as a reasonable analog of the functional physical behavior being modeled, which is the core of finite element method.

The software COMSOL is used to generate the grid. If using coarse mesh size (CMS) to structure the grid, the accuracy of calculation results is not enough because of the less grids. But too refined meshing grid takes a lot of memory which leads to long time calculation. All things considered, this paper used normal mesh size (NMS) that the number of generated elements is between coarse mesh size (CMS) and fine mesh size (FMS) to create the meshing grid as shown in Fig.5 and Fig.7.

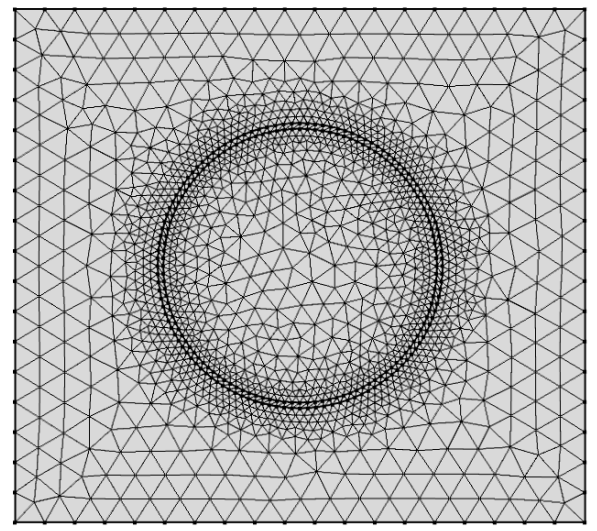

Fig.5. Meshing grid of single cell model (3014 elements)

\subsection{Multi-cell model}

Because of the special electrical properties of cell membranes, cells show different characteristics in the electric field with different frequencies and the changes of cell membrane's electrical properties lead to changes on physiological state of cells.

In order to observe the dielectric properties of cells under different physiological states, this paper established a multi-cell model based on the single cell model in section 2.1 as shown in Fig.6(a) and the meshing grid using SMM is shown in Fig.6(b).

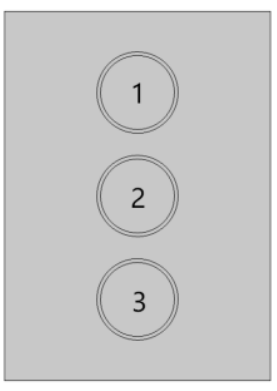

(a)

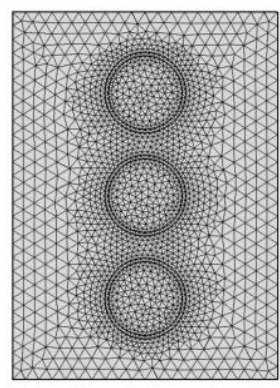

(b)
Fig.6(a). Geometric model of multi-cell

(b) Meshing grid of multi-cell model (2962 elements)

And three simulation works were implemented to watch the influence on cells that caused by the changes on the membrane electrical parameters. Table. 1 shows the parameters setting of three groups of experiments. The parameters are set as the single cell model in Fig.4.

Table.1. Membrane electrical parameters of multi-cell model

\begin{tabular}{|c|c|c|c|c|c|c|}
\hline & \multicolumn{2}{|c|}{ Group A } & \multicolumn{2}{|c|}{ Group B } & \multicolumn{2}{|c|}{ Group C } \\
\hline & $\varepsilon_{\mathrm{m}}$ & $\begin{array}{c}\sigma_{\mathrm{m}} \\
(\mathrm{S} / \mathrm{m})\end{array}$ & $\varepsilon_{\mathrm{m}}$ & $\begin{array}{c}\sigma_{\mathrm{m}} \\
(\mathrm{S} / \mathrm{m})\end{array}$ & $\varepsilon_{\mathrm{m}}$ & $\begin{array}{c}\sigma_{\mathrm{m}} \\
(\mathrm{S} / \mathrm{m})\end{array}$ \\
\hline 1 & \multirow{3}{*}{5} & $10^{-2}$ & 3 & \multirow{3}{*}{$10^{-5}$} & 3 & $10^{-8}$ \\
\hline 2 & & $10^{-5}$ & 5 & & 5 & $10^{-5}$ \\
\hline 3 & & $10^{-8}$ & 7 & & 7 & $10^{-2}$ \\
\hline
\end{tabular}

\section{Simulation result}

\subsection{Single cell simulation result}

The current density distribution at three typical different frequencies $(1 \mathrm{MHz}, 50 \mathrm{MHz}$ and $100 \mathrm{MHz}$ separately) as shown in Fig.8. It can be observed that there is no current flowing through the cell model at $1 \mathrm{MHz}$. It is because the membrane works as capacitance in parallel with a large resistance. The membrane has high insulation and low conductivity at low frequency. With the frequency gradually increasing, a part of current starts to pass through the membrane at frequency 50MHz. It's apparent that the cell membrane becomes electrically invisible when the frequency reaches $100 \mathrm{MHz}$. The current density in the whole domain is homogeneous.
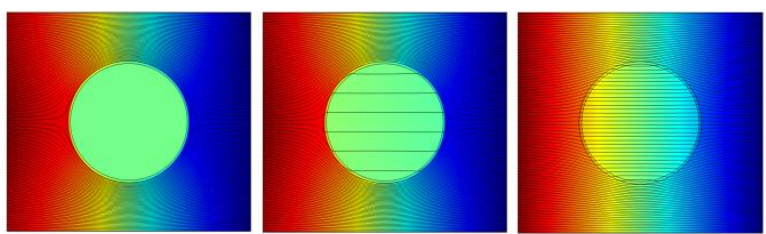
$1 \mathrm{MHz}$

$50 \mathrm{MHz}$

$100 \mathrm{MHz}$

Fig.8.Current density distribution of single cell at different frequency

\subsection{Multi-cell simulation result}

Table.2. Multi-cell model simulation results

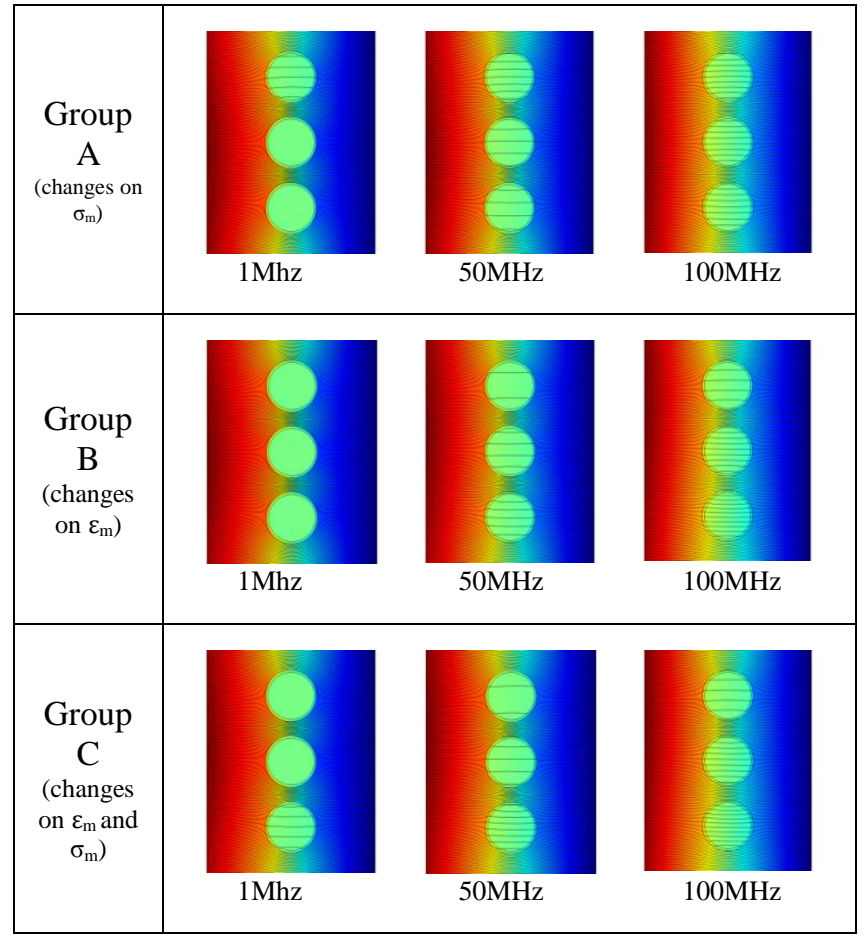

Table 2 shows the three sets of simulation results, in the same current field, the three cells with different membrane have respective frequency response. This because the membrane works as a capacitance in parallel with a large resistance, when setting different electrical parameters (permittivity $\varepsilon$ and conductivity $\sigma$ ) to the membrane, the value of the capacitance and the resistance changed. The simulation results proves that the change of cell's physiological status can affect its dielectric properties on account of changes on membrane.

\section{Discussion}

The finite element method has been widely used in mechanics of biological tissue ${ }^{[6]}$. But at the cell level, there are few papers that introduce the electromagnetic characteristics $^{[7]}$.

This paper preliminarily build a single-cell model and a multi-cell model from microcosmic view and conduct a tentative research at the dielectric properties of the biological cell with finite element method. In view of physiology, most diseases are originated from cell lesion, and the dielectricity of cell is changed earlier than the structural lesion. If the cell dielectricity can be detected by current signal at routine check-up, it's worthwhile for diseases precaution.

The search results of this paper indicate that the different physiology status cells can show up different dielectrical characteristics when is stimulated with different frequencies.

\section{Acknowledgements}

This research work is supported by the National Natural Science Foundation of China (Grant No.61301246) and the Science and Technology Project of Tianjin (12JCYBJC19300).

\section{References}

1. RJ Yerworth, RH Bayford, G Cusick, M Conway, DS Holder, Design and performance of the UCLH Mark 1b 64 channel electrical impedance tomography (EIT) system, optimized for imaging brain function, Physiol Meas, 2002,23: 149-158.

2. XY Chen, XQ Lv, Meng Du, Experimental verification of depolarization effects in bioelectrical impedance measurement, Bio-Medical Materials and Engineering, 2014,24: 3675-3683.

3. JR Wang, BY Sun, HX Wang, Qing Sun, Experimental Study of Dielectric Properties of Human Lung Tissue in Vitro, Journal of Medical and Biological Engineering, 2014, 34(6): 598-604.

4. Jiaming Jin, The finite element method in electromagnetics, John\&Sons, 2014.

5. K Asami, Dielectric dispersion in biological cells of complex geometry simulated by the three-dimension finite difference method, Journal of Physics D: Applied Physics, 2006, 39(3): 492.

6. A Tchako, A M Sadeyh, Stress Changes in intervertebral discs of the leruical spine due to partial discectomies of and fusion, Journal of Biomechanical Engineering, 2009, 131(5):1-11.

7. C Ding, X Liu, P Shang, Progress of the technology and application of dielectric spectroscopy of cell suspensions, Beijing Biomedical Engineering, 2014, 33(1):94-99. 